\title{
COMBINING FEATURE FUSION AND DECISION FUSION FOR CLASSIFICATION OF HYPERSPECTRAL AND LIDAR DATA
}

\author{
Wenzhi Liao, Rik Bellens, Aleksandra Pižurica, Sidharta Gautama, Wilfried Philips \\ Ghent University-TELIN-IPI-iMinds, Sint-Pietersnieuwstraat 41, B-9000 Ghent, Belgium
}

\begin{abstract}
This paper proposes a method to combine feature fusion and decision fusion together for multi-sensor data classification. First, morphological features which contain elevation and spatial information, are generated on both LiDAR data and the first few principal components (PCs) of original hyperspectral (HS) image. We got the fused features by projecting the spectral (original HS image), spatial and elevation features onto a lower subspace through a graph-based feature fusion method. Then, we got four classification maps by using spectral features, spatial features, elevation features and the graph fused features individually as input of SVM classifier. The final classification map was obtained by fusing the four classification maps through the weighted majority voting. Experimental results on fusion of HS and LiDAR data from the 2013 IEEE GRSS Data Fusion Contest demonstrate effectiveness of the proposed method. Compared to the methods using single data source or only feature fusion, with the proposed method, overall classification accuracies were improved by $10 \%$ and $2 \%$, respectively.
\end{abstract}

Index Terms - Data fusion, remote sensing, hyperspectral image, LiDAR data, graph-based

\section{INTRODUCTION}

Nowadays, advanced sensor technologies allow us to measure different aspects of the objects on the Earth's surface, from spectral characteristics in hyperspectral (HS) images, to height information in Light Detection And Ranging (LiDAR) data. It is clear that HS or LiDAR data alone might not be sufficient to obtain reliable classification results in a complex urban context. Instead, the combination of both data sources can contribute to a more comprehensive interpretation of the ground objects. For example, spectral signatures cannot differentiate between objects made of the same material (e.g. roofs and roads made with the same asphalt), while the latter may be easily distinguished by their altitude. On the other hand, LiDAR data alone may fail to discriminate between objects that are quite different in nature (e.g. grass field and swimming pool), but similar in their altitude.

Due to the increased availability of HS and LiDAR data from the same area, the fusion of these remote sensing data

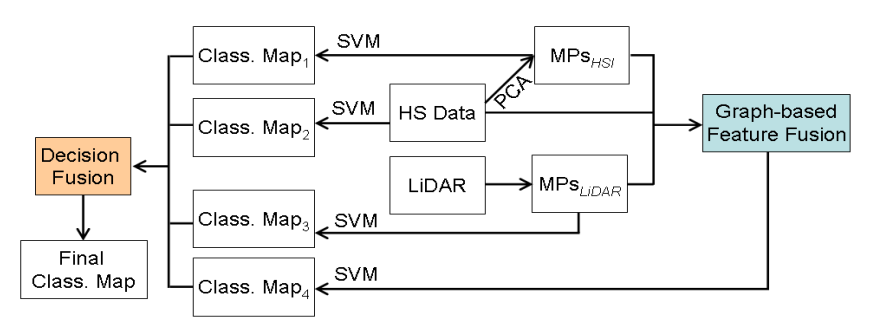

Fig. 1: Proposed data fusion framework.

have been of great interest for many practical applications [1.3. 4]. Lemp and Weidner [1] exploit HS and LiDAR data for the classification of urban areas, using LiDAR for the segmentation of the scene, and then HS data for the classification of the resulting regions. The joint use of HS and LiDAR remote sensing data for the classification of complex forest areas was investigated in [3]. They proposed a novel classification system, based on different possible classifiers that were able to properly integrate multi-sensor information. The very recent method [4] applied morphological attribute profiles (EAPs) [5] to both HS and LiDAR data for a classification task. Their method jointly considered the features extracted by EAPs computed on both HS and LiDAR data, and fused spectral, spatial and elevation information in a stacked architecture. However, stacking too many features from different data sources may lead to the problem of the curse of dimensionality and excessive computation time [2]. Furthermore, the performances by simply stacking several feature sources together are not always better than using single feature source. This is because the information contained by different feature sources is not equally represented or measured. Our previous approach overcame these problems by fusing multi-sensor data through a fusion graph [6], and won the "Best Paper Challenge" of the 2013 IEEE GRSS Data Fusion Contest.

In this paper, we propose a method to combine feature fusion and decision fusion together for multi-sensor data classification in Fig 1. First, morphological features which contain elevation and spatial information, are generated on both $\mathrm{Li}$ DAR data and the first few principal components (PCs) of original HS image. We got the fused features by projecting the spectral, spatial and elevation features onto a lower subspace through our graph-based feature fusion method [6]. 
Then, we used spectral features, spatial features, elevation features and the graph fused features (GFF) individually as inputs of SVM classifier to generate four classification maps. The final classification map was obtained by fusing all the classification maps through the weighted majority voting [7].

\section{METHODOLOGY}

\subsection{Morphological features of HS image and LiDAR data}

Morphological features are generated by either applying morphological openings or closings by reconstruction on the image, using a structural element (SE) of predefined size and shape. For example, the morphological profile (MP) with disk SE carry information about the minimum size of objects, whereas directional MP indicate of the maximum size of objects [8]. An opening acts on bright objects (for LiDAR data, the bright regions are actually areas with the high elevation, such as the top of the roof) compared with their surrounding, while closings act on dark (low height in the LiDAR data) objects. For example, an opening deletes bright objects that are smaller than the SE. By increasing the size of the SE and repeating the previous operation, a complete morphological profile (MP) is built, carrying information about the size and the shape of objects in the image.

In our experiments, morphological features are generated by applying morphological openings and closings with partial reconstruction on both LiDAR data and the first 2 principal components (PCs) (representing more than 99\% of the cumulative variance) of original HS image. The effect of using morphological features with partial reconstruction for classification of remote sensing data from urban areas has been discussed in our previous work [8, 10]. For disk-shaped SE, MPs with 15 openings and closings (ranging from 1 to 15 with step size increment of 1) are computed for both LiDAR data and the first 2 PCs of HS image. For linear structuring elements, MPs with 20 openings and closings (ranging from 5 to 100 with step size increment of 5) are constructed for both LiDAR data and the first 2 PCs of HS image.

\subsection{Graph-based Feature Fusion Method}

Using single data source may not be sufficient for a reliable classification, but combining many of them can lead to problems like the curse of dimensionality, excessive computation time and so on. Before fusing all the feature sources, we normalized their dimensions and reduce the noise throughout the given feature space with Kernel Principal Component Analysis (KPCA) [9, 10], the same as we did in [6]. We assume the dimension of each feature source is already normalized to the smallest dimension of all the feature sources $D=70$. Let $\mathbf{X}^{S p e}=\left\{\mathbf{x}_{i}^{S p e}\right\}_{i=1}^{n}, \mathbf{X}^{S p a}=\left\{\mathbf{x}_{i}^{S p a}\right\}_{i=1}^{n}$ and $\mathbf{X}^{\text {Ele }}=$ $\left\{\mathbf{x}_{i}^{\text {Ele }}\right\}_{i=1}^{n}$, where $\mathbf{x}_{i}^{\text {Spe }} \in \Re^{D}, \mathbf{x}_{i}^{S p a} \in \Re^{D}$ and $\mathbf{x}_{i}^{\text {Ele }} \in$ $\Re^{D}$ denote the dimensional normalized features of spectral, spatial and elevation, respectively. $\mathbf{X}^{S t a}=\left\{\mathbf{x}_{i}^{S t a}\right\}_{i=1}^{n}=$ $\left[\mathbf{X}^{\text {Spe }} ; \mathbf{X}^{\text {Spa }} ; \mathbf{X}^{\text {Ele }}\right]$, and $\mathbf{x}_{i}^{\text {Sta }}=\left[\mathbf{x}_{i}^{\text {Spe }} ; \mathbf{x}_{i}^{\text {Spa }} ; \mathbf{x}_{i}^{\text {Ele }}\right] \in \Re^{3 D}$ denotes the vector stacked by the features of spectral, spatial and altitude.

We first build a graph for each feature source, for example, the graph constructed by spectral features (i.e., $\mathbf{G}^{\text {Spe }}=$ $\left(\mathbf{X}^{S p e}, \mathbf{A}^{S p e}\right)$ ), where $\mathbf{A}^{S p e}$ represents the edges of the graph. The edge between data point $\mathbf{x}_{i}^{S p e}$ and $\mathbf{x}_{j}^{S p e}$ is here denoted as $A_{i j}^{S p e} \in\{0,1\} ; A_{i j}^{S p e}=1$ if $\mathbf{x}_{i}^{S p e}$ and $\mathbf{x}_{j}^{S p e}$ are "close" and $\mathbf{x}_{i}^{S p e}$ and $\mathbf{x}_{j}^{S p e}$ are "far apart". The "close" is defined as belonging to $k$ nearest neighbors $(k \mathrm{NN})$ of the other data points. The $k$ NNs of the data point $\mathbf{x}_{i}^{S p e}$ are its $k$ nearest neighbors in terms of spectral signatures. On the other hand, when the graph is constructed by spatial features, the $k \mathrm{NNs}$ of the data point $\mathbf{x}_{i}^{S p a}$ are its $k$ nearest neighbors in terms of spatial characteristics; whereas the $k \mathrm{NNs}$ of $\mathbf{x}_{i}^{E l e}$ are its $k$ nearest neighbors in terms of elevation characteristics. We define a fusion graph $\mathbf{G}^{F u s}=\left(\mathbf{X}^{S t a}, \mathbf{A}^{F u s}\right)$, where:

$$
\mathbf{A}^{F u s}=\mathbf{A}^{S p e} \odot \mathbf{A}^{S p a} \odot \mathbf{A}^{E l e}
$$

The operator ' $\odot$ ' denotes element-by-element multiplication, i.e. $A_{i, j}^{F u s}=A_{i, j}^{S p e} * A_{i, j}^{S p a} * A_{i, j}^{\text {Ele }}$. This means the stacked data points $\mathbf{x}_{i}^{S t a}$ and $\mathbf{x}_{j}^{S t a}$ are connected only if they have similar on all spectral, spatial and elevation characteristics, for more details to obtain the fused features, we refer the readers to our recent work [6].

\subsection{Decision Fusion-based classification}

Fig. 1 shows the framework of our proposed decision fusion. After fusing spectral, spatial and elevation features on feature level, we use single feature source (spectral, spatial and elevation feature individually) and the fused features independently as input of SVM classifiers to generate four classification maps. In real applications, it is impossible to get a fully certain and complete classification map. Therefore it is necessary to weight each of the outputs (classification maps) so that the final ensemble reflects our knowledge of the reliability. In other words, one can assign different weights to different outputs in order to achieve a more satisfactory final classification map. One simple way to adjust the contribution of each output is to apply the weighted majority voting [7]. The choice of classifiers weighting factors will have a significant effect on the results of the classification because the contribution of each output will be reduced or enhanced in proportion to its weight. This paper employs overall classification accuracy to measure the classifiers weighting parameters.

\section{EXPERIMENTAL RESULTS}

Experiments are done on a hyperspectral image and a LiDAR data which were acquired by the NSF-funded Center for Airborne Laser Mapping (NCALM) [11] on June 2012 over 
the University of Houston campus and the neighboring urban area. The hyperspectral imagery has 144 spectral bands with wavelength range from $380 \mathrm{~nm}$ to $1050 \mathrm{~nm}$. Both the data have the same spatial resolution $(2.5 \mathrm{~m})$. The whole scene of both the data, consisting of the full $349 \times 1905$ pixels, contains 15 classes, Fig. 2 shows an RGB composition.

The SVM classifier with radial basis function (RBF) kernels is applied in our experiments. The parameters of SVM classifier are set the same as in our previous work [6]. We compare our proposed methods with the schemes of (1) Only using spectral information (Original HSI); (2) Using the MPs computed on the first 2 PCs of HS image (MPs $\left.{ }_{H S I}\right)$; (3) Using the MPs computed on the LiDAR data $\left(\operatorname{MPs}_{L i D A R}\right)$; (4) Using the features fused by using our previous method in [6]. The classification results are quantitatively evaluated by measuring the Overall Accuracy (OA), the Average Accuracy (AA) and the Kappa coefficient $(\kappa)$. Table 1 shows the accuracies values obtained from the experiments, Fig. 2 shows the classification maps.

It can be found that using single feature source is not sufficient for a reliable classification. Each feature source has its advantages on different classes. For examples, spectral features are good to discriminate some natural materials such as grass and tree, while spatial and elevation features are better on some man-made objects, of which LiDAR are better on some big objects with similar elevation (e.g. commercial and highway). Our previous method couple dimension reduction and data fusion together, with higher accuracies but less features than using single feature source. However, we can see that some classes from using the fused features have lower accuracies even than using single feature source, such as 'Grass Healthy' and 'Road'. By combining feature fusion and decision fusion, our proposed method performs better than the other schemes, with $10.14 \%-23.18 \%$ and $2.27 \%$ improvements in OA than using single feature source and only feature fusion, respectively.

\section{CONCLUSION}

The contribution of this paper is a methodology to combine feature fusion and decision fusion together for the multisensor data classification. The morphological features with partial reconstruction, which carry the spatial and elevation information, are first generated on both the first PCs of HS image and LiDAR data. We got the fused features by projecting the spectral, spatial and elevation features onto a lower subspace through a graph-based feature fusion method. Then, we got four classification maps by using spectral features, spatial features, elevation features and the graph fused features individually as inputs of SVM classifier. We generate the final classification map by fusing the four classification maps through the weighted majority voting. Experimental results on the classification of the real HS and LiDAR data show the efficiency of the proposed method.

\section{Acknowledgment}

This work was financially supported by the SBO-IWT project Chameleon: Domain-specific Hyperspectral Imaging Systems for Relevant Industrial Applications. The authors would like to thank the Hyperspectral Image Analysis group and the NSF Funded Center for Airborne Laser Mapping (NCALM) at the University of Houston for providing the data sets used in this study, and the IEEE GRSS Data Fusion Technical Committee for organizing the 2013 Data Fusion Contest.

\section{REFERENCES}

[1] D. Lemp and U. Weidner, "Improvements of roof surface classification using hyperspectral and laser scanning data," in Proc. ISPRS Joint Conf.: 3rd Int. Symp. Remote Sens. Data Fusion Over Urban Areas (URBAN), 5th Int. Symp. Remote Sens. Urban Areas (URS), Tempe, AZ, Mar. 14-16, 2005.

[2] G. F. Hughes, "On the mean accuracy of statistical pattern recognizers," IEEE Transactions on Information Theory, vol. 14, no. 1, pp. 55-63, 1968.

[3] M. Dalponte, L. Bruzzone, D. Gianelle, "Fusion of Hyperspectral and LIDAR Remote Sensing Data for Classification of Complex Forest Areas," IEEE Trans. Geosci. Remote Sens., vol. 46, no. 5, pp. 1416-1427, May 2008.

[4] M. Pedergnana, P. Reddy Marpu, M. Dalla Mura, J. A. Benediktsson, L. Bruzzone, "Classification of Remote Sensing Optical and LiDAR Data Using Extended Attribute Profiles," IEEE Journals on Selected Topics in Signal Processing, vol. 6, no. 7, pp. 856-865, Nov. 2012.

[5] M. Dalla Mura, J. A. Benediktsson, B. Waske, and L. Bruzzone, "Extended profiles with morphological attribute filters for the analysis of hyperspectral data," Int. J. Remote Sens., vol. 31, no. 22, pp. 5975-5991, Nov. 2010.

[6] C. Debes, A. Merentitis, R. Heremans, J. Hahn, N. Frangiadakis, T. Kasteren, W. Liao, R. Bellens, A. Pizurica, S. Gautama, W. Philips, S. Prasad, Q. Du, F. Pacifici, "Hyperspectral and LiDAR Data Fusion: Outcome of the 2013 GRSS Data Fusion Contest," IEEE Journal of Selected Topics in Applied Earth Observations and Remote Sensing, 2014 (In Press).

[7] N. Littlestone and M. Warmuth, "The weighted majority algorithm," Information and Computation, vol. 108, no. 2, pp. 212-261, 1994.

[8] W. Liao, R. Bellens, A. Pižurica, W. Philips and Y. Pi, "Classification of Hyperspectral Data Over Urban Areas Using Directional Morphological Profiles and SemiSupervised Feature Extraction," IEEE Journal of Selected Topics in Applied Earth Observations and Remote Sensing, vol. 5, no. 4, pp. 1177-1190, Aug. 2012. 
Table 1: Classification accuracies obtained by the described schemes.

\begin{tabular}{|l|c|c|c|c|c|}
\hline & Original HSI & MPs $_{H S I}$ & MPs $_{L i D A R}$ & GFF & Proposed \\
\hline No. of Features & 144 & 140 & 70 & 26 & 4 \\
\hline OA (\%) & 80.72 & 82.43 & 69.39 & 90.30 & $\mathbf{9 2 . 5 7}$ \\
AA (\%) & 83.40 & 84.99 & 68.42 & 91.30 & $\mathbf{9 3 . 0 8}$ \\
$\kappa(\%)$ & 79.23 & 81.02 & 66.79 & 89.48 & $\mathbf{9 1 . 9 4}$ \\
\hline Grass Healthy & $\mathbf{8 2 . 1 5}$ & 80.25 & 35.61 & 73.31 & 81.77 \\
Grass Stressed & 81.58 & 80.64 & 67.11 & $\mathbf{9 7 . 8 4}$ & $\mathbf{9 7 . 8 4}$ \\
Grass Synthetis & 99.80 & $\mathbf{1 0 0 . 0 0}$ & 79.60 & $\mathbf{1 0 0 . 0 0}$ & $\mathbf{1 0 0 . 0 0}$ \\
Tree & 92.80 & 84.09 & 72.92 & $\mathbf{9 7 . 8 2}$ & 97.73 \\
Soil & 97.92 & $\mathbf{1 0 0 . 0 0}$ & 83.52 & 99.24 & 99.72 \\
Water & 95.10 & 95.10 & 66.43 & $\mathbf{9 9 . 3 0}$ & $\mathbf{9 9 . 3 0}$ \\
Residential & 76.21 & 87.31 & 76.59 & 88.15 & $\mathbf{8 9 . 0 9}$ \\
Commercial & 54.51 & 45.58 & 91.45 & $\mathbf{9 6 . 2 0}$ & 93.83 \\
Road & 78.47 & $\mathbf{9 1 . 0 3}$ & 59.21 & 86.59 & 88.10 \\
Gighway & 60.04 & 60.42 & 64.86 & 76.83 & $\mathbf{8 1 . 7 6}$ \\
Railway & 79.51 & 87.10 & 88.24 & 92.41 & $\mathbf{9 6 . 1 1}$ \\
Parking Lot 1 & 82.90 & 86.84 & 70.89 & 85.69 & $\mathbf{9 4 . 5 2}$ \\
Parking Lot 2 & 72.63 & 76.49 & 55.09 & 76.49 & $\mathbf{7 6 . 8 4}$ \\
Tennis Court & $\mathbf{1 0 0 . 0 0}$ & $\mathbf{1 0 0 . 0 0}$ & $\mathbf{1 0 0 . 0 0}$ & $\mathbf{1 0 0 . 0 0}$ & $\mathbf{1 0 0 . 0 0}$ \\
Running Track & 97.25 & $\mathbf{1 0 0 . 0 0}$ & 14.80 & 99.58 & 99.58 \\
\hline
\end{tabular}

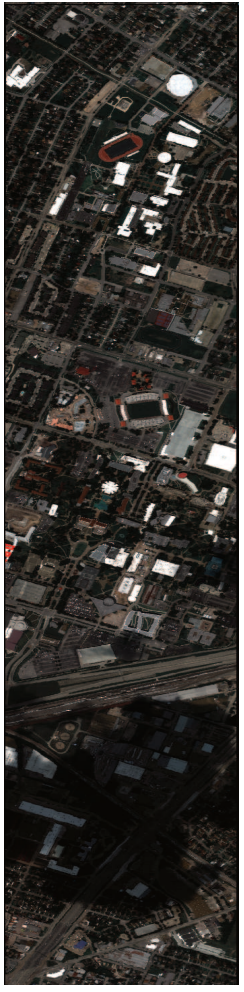

(a)

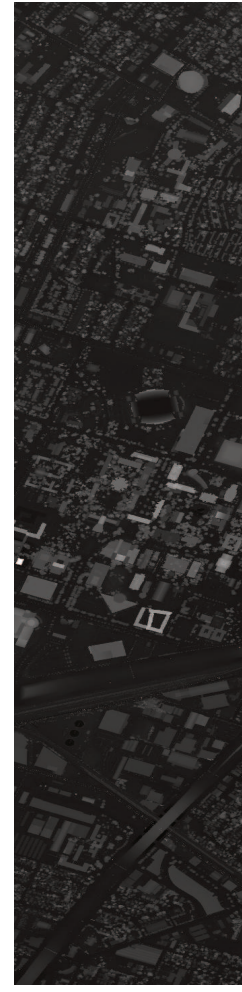

(b)

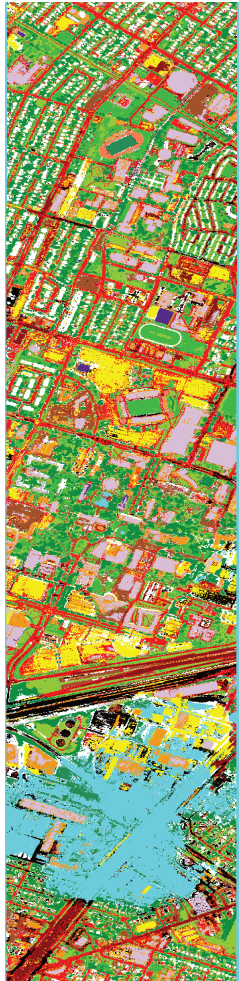

(c)

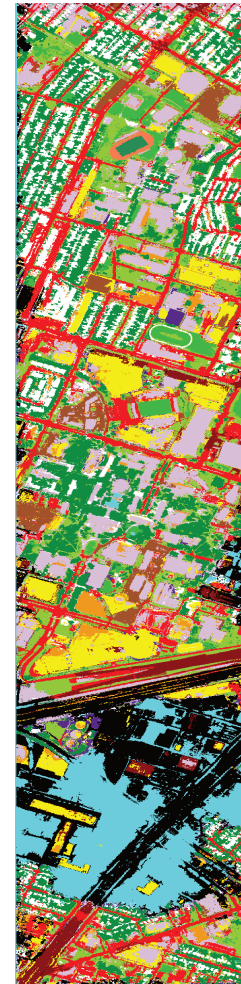

(d)

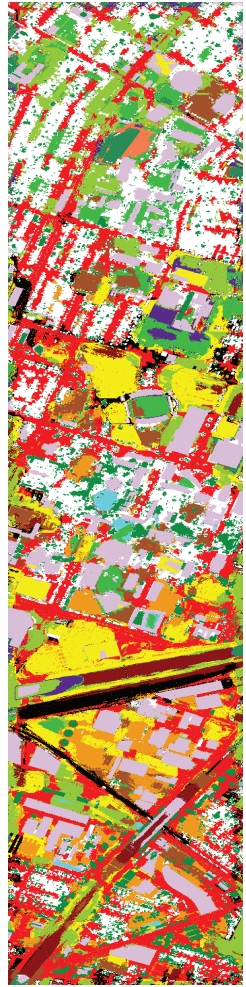

(e)

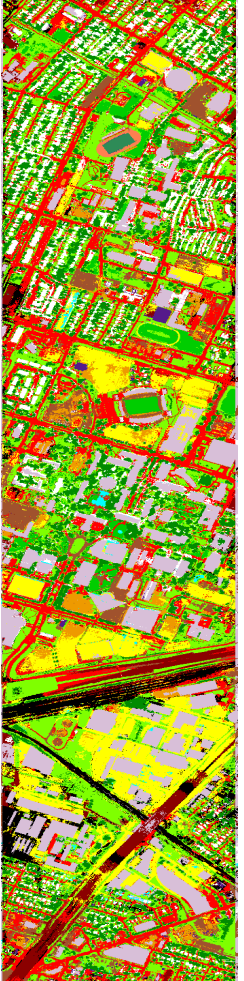

(f)

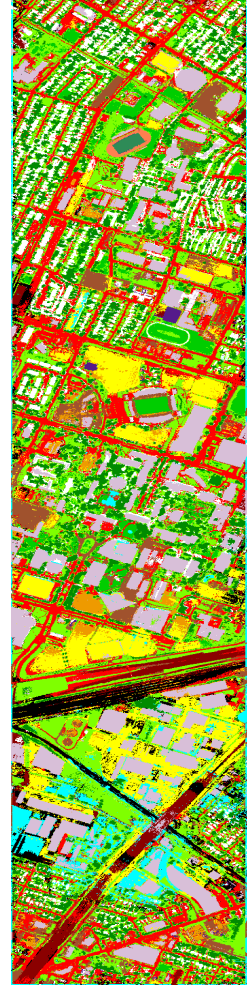

(g)

Fig. 2: Classification maps produced by the described schemes. (a) False color composite image of HS image; (b) LiDAR image; and Thematic map of using (c) Original HS data; (d) MPs of HS data; (e) MPs of LiDAR data; (f) Graph-based feature fusion [6]; (g) Proposed method.

[9] B. Scholkopf, A.J. Smola and K.R. Muller, "Nonlinear component analysis as a kernel eigenvalue problem," Neural Computation, vol. 10, pp. 1299-1319, 1998.

[10] W. Liao, R. Bellens, A. Pižurica, W. Philips, Y. Pi, "Classification of Hyperspectral Data over Urban Areas based on Extended Morphological Profile with Partial
Reconstruction", In Proc. Advanced Concepts for Intelligent Vision Systems (ACIVS) 2012, Brno, Czech Republic, pp. 278-289, 2012.

[11] 2013 IEEE GRSS Data Fusion Contest, Online: http://www.grss-ieee.org/community/technicalcommittees/data-fusion/. 